\title{
Cascading failure analysis of power flow on wind power based on complex network theory
}

\author{
Yushu SUN, Xisheng TANG ( $\square)$
}

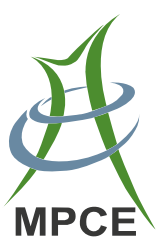

\begin{abstract}
Cascading failure is a potential threat in power systems with the scale development of wind power, especially for the large-scale grid-connected and long distance transmission wind power base in China. This introduces a complex network theory (CNT) for cascading failure analysis considering wind farm integration. A cascading failure power flow analysis model for complex power networks is established with improved network topology principles and methods. The network load and boundary conditions are determined to reflect the operational states of power systems. Three typical network evaluation indicators are used to evaluate the topology characteristics of power network before and after malfunction including connectivity level, global effective performance and percentage of load loss (PLL). The impacts of node removal, grid current tolerance capability, wind power instantaneous penetrations, and wind farm coupling points on the power grid are analyzed based on the IEEE 30 bus system. Through the simulation analysis, the occurrence mechanism and main influence factors of cascading failure are determined. Finally, corresponding defense strategies are proposed to reduce the hazards of cascading failure in power systems.
\end{abstract}

Keywords Complex network theory (CNT), Cascading failure, Wind power, Power flow analysis model, Defense strategies

CrossCheck date: 21 November 2014

Received: 20 October 2014/ Accepted: 26 November 2014/

Published online: 11 December 2014

(C) The Author(s) 2014. This article is published with open access at Springerlink.com

Y. SUN, X. TANG, Institute of Electrical Engineering, Chinese Academy of Sciences, Beijing, China

$(\bowtie)$ e-mail: tang@mail.iee.ac.cn

Y. SUN

e-mail: yushusun@mail.iee.ac.cn

\section{Introduction}

It is proved that almost all the blackouts are caused by cascading failures, which are triggered initially by single or multiple disturbances in certain circumstances, such as long time overload or stability issues in bulk power system [1]. For example, the " $8 \cdot 14$ " blackout in North America in 2003 was induced by five $345 \mathrm{kV}$ AEP-DPL ground fault lines in MISO. Then, the transmission line cascading trips took place to make the failure propagation, and eventually power outage occurred $[2,3]$.

With the rapid development of wind power around the world, its penetration in the power grid grows higher and higher. Different from the distributed utilization of wind power in North America and Europe, large-scale gridconnected and long distance transmission of wind power, also known as wind power base (WPB), is inevitable in China due to the uneven distribution of wind resources and load centers. The intermittent and stochastic of output power of wind farms, as well their easy tripping under abnormal conditions, will increase the occurrence probability of cascading failures in power systems.

Recently, several typical methods on cascading failure have been researched [4-7], including pattern search, macroscopic evaluation, complex system methods and complex network theory. Heuristic search is often used by the pattern search method. However, it is hard to use practically due to the quite low search precision and efficiency. The research object of macroscopic evaluation method is based on sample data, so the evaluation is not accurate enough and lack of universality. The model for complex systems just reveals the mechanism of cascading failures qualitatively, which are hard to describe the details of an actual power system. From the perspective of network, CNT can be used to analyze the impacts of power grid topology structure on cascading failure, which provides a new perspective and method. CNT has 
been widely used in power system cascading failure analysis [8-11].

Complex network is a complicated system, which contains a high number of individuals and the interaction among them. A typical network can be expressed as many nodes and edges, where every node represents individual and every edge stands for the mutual relation of two individuals. For power system, power plants, substations (or buses) and consumers can be considered to be the nodes, and the transmission lines are used as the edges. Then, the basic topology of complex power system will be acquired. CNT is used to study the structure characteristics of power system and dynamic propagation behaviors of failure. Its purpose is to explain the cause and development law of power blackout, and then effective measures and suggestions can be proposed to prevent the occurrence of power accident. The main feature of CNT is that the analysis model is easy to be built, and can reflect internal relations and operating characteristics of the system. In addition, it has good application flexibility and adaptability for power system analysis.

In general, the cascading failure analysis based on CNT can be classified into the following steps, including load definition, boundary condition determination, failure mode selection, network failure evaluation and corresponding defense strategy. As to the definition of load, the node load is defined primarily by the total number of the shortest paths that pass through the node, i.e., node betweenness [12-17]. Wang and Rong defined the relevant functions of the node degree as the node load [18-21]. But the above load definitions lose sight of the electrical specification of the grid [22] and power transmission doesn't follow the Kirchhoff's Law. In addition, Bompard and Xu et al. used the extended betweenness [23-25] or electrical betweenness [26-29] based on power transfer distribution factor (PTDF) as the load in the power grid. However, the extended or electrical betweenness identifies the criticality of lines from a structural point of view without considering operational states of power system. As to boundary conditions, the capacity $C_{i}=(1+\alpha) L_{i}$ of node $i$ is supposed proportional to its initial load $L_{i}$ [12-17], where $\alpha(>0)$ is the tolerance parameter. However, the upper limit of the capacity is considered while the lower limit is often ignored in simulation. As to failure mode selection, removing special nodes or edges is often adopted in traditional grid failure analysis [30, 31]. But the type of failure becomes diversification due to wind power integration. As to network failure evaluation, many of the existing evaluation indicators [28, 32, 33] are just suitable for general complex networks which can not accurately reflect electrical characteristics before and after malfunction in power system. As to defense strategy,many measures are provided to suppress cascading failure [34-36] in previous researches, but the ones which are suitable for wind power integration should be proposed.

In this paper, a power system cascading failure power flow analysis model based on CNT is established to study the influence of WPB on power system cascading failure. In the model, power flow is used as the load to exactly describe the distribution of voltage and power in power grid. The upper and lower operating limits of power system are both considered under condition of wind power integration. Three typical evaluation indicators including connectivity level, global effective performance and percentage of load loss (PLL) are presented to evaluate network performance before and after malfunction. Accordingly, some effective defense strategies are proposed to reduce the impacts of wind power on the stability and the security of power system.

The studies indicate that influences of wind power integration on power system cascading failure are analyzed, and the harm extent is quantified compared with traditional fault. In the different simulation scenarios, the dominant factors of cascading failure caused by wind power are determined, and then the corresponding defensive strategies are put forward. It provides a reference for the prevention and control of grid-connected wind power system cascading failure, and promotes the rapid development of large-scale grid-connected wind power.

The remains of this paper are organized as follows. Section 2 introduces the topology principles and basic characteristics of CNT considering wind power integration. The cascading failure analysis model is presented in Section 3. In Section 4, the influences of node removal, grid current tolerance capability, different wind power instantaneous penetrations and wind farms coupling points on power grid are explored through a case study. Conclusions are given in Section 5.

\section{Complex network theory with wind power integration}

\subsection{Topology principles and methods}

CNT method can reflect the network topology, operating characteristics and other elements of power grid from the static and dynamic perspective. In order to analyze the characteristics of the grid accurately, a grid-connected wind power system directed-weighted network model based on undirected-unweighted and undirected-weighted network model [37-39] is established.

1) Only the high-voltage transmission grids are considered, while the internal main wirings of power plants, substations and distribution network are ignored. 
2) The power plants, buses and customers are considered as generation nodes, transmission nodes and load nodes, respectively. They have their own characteristic parameters and voltage level. Wind power is added to the traditional grid, which is equivalent to PQ node to distinct from conventional power generation node.

3) The weight of power generation node is defined as the rated output of the generator. The maximum load demand is considered as the weight of load node. And the weight of the transmission node is equivalent to its capacity.

4) The network edges are denoted as the branches of the transmission lines and transformers, the weight as the impedance of the line and the direction as that of power flow.

5) The transmission lines connected the same two nodes are merged, and the branch-path of the parallel capacitors are neglected. In order to make the network structure diagram simplification, self-loop and excessive edges are eliminated in the grid.

Through the above mapping process, the power grid can be modeled as a weighted, directed and sparse connected graph with $N$ nodes and $K$ edges.

\subsection{Basic characteristics}

1) Node degree and its distribution

The degree of a node is the total number of the edges attached to it, usually represented by $k$. Degree distribution is the ratio of nodes in the network with degree $k$. The degree of nodes can measure importance of nodes in the grid, while degree distribution reflects the uniformity of the network.

\section{2) Clustering coefficient}

Assuming that node $i$ has degree $k_{i}$, and there are $t_{i}$ edges among its nearest nodes. For the nearest nodes, $k_{i}$ $\left(k_{i}-1\right) / 2$ edges can be acquired under the condition of a complete graph. The clustering coefficient of node $i$ can be defined as

$\gamma_{i}=\frac{t_{i}}{k_{i}\left(k_{i}-1\right) / 2}$

Clustering coefficient indicates the clustering and tightness of nodes in the grid.

\section{3) Electrical distance}

Electrical distance is the electric potential difference caused by a unit of current transmitted from node $i$ to node $j$, namely, is equivalent impedance between two nodes.

$d_{i j}=\left|Z_{i i}+Z_{j j}-2 Z_{i j}\right|$ where $Z_{i i}$ and $Z_{j j}$ are the self-impedances of node $i$ and $j$, respectively; $Z_{i j}$ is the mutual impedance between node $i$ and $j$. The size of the electrical distance reflects electrical connection between different nodes in the power grid.

\section{4) Electrical betweenness}

According to the power flow transmission characteristics, the electrical betweenness of edge $(m, n)$ is defined as:

$B_{e}(m, n)=\sum_{i \in G, j \in L} w_{i j}\left|I_{m n}(i, j)\right|$

where $G$ and $L$ are the sets of generation nodes and load nodes, respectively; $w_{i j}$ are the weights of power transmitted from node $i$ to $j ; I_{m n}(i, j)$ is the generated currents in the edges $(m, n)$, when a unit of current is transmitted from the generation node $i$ to the load node $j$. In addition, $I_{m n}(i, j)$ are also known as the power transfer distribution factors (PTDFs) to reflect the sensitivity of the power flowing in each line for a power injection/withdrawal at a couple of nodes.

The electrical betweenness of node $k$ can be calculated as:

$B_{n}(k)= \begin{cases}\frac{1}{2}\left(\sum_{l \in F(k)} B_{e}(k, l)+\sum_{j \in L} w_{k j}\right) & k \in G \\ \frac{1}{2}\left(\sum_{l \in F(k)} B_{e}(k, l)+\sum_{j \in G} w_{k j}\right) & k \in L \\ \frac{1}{2} \sum_{l \in F(k)} B_{e}(k, l) & k \notin G, k \notin L\end{cases}$

where $F(k)$ is the set of nodes that are connected to node $k$.

Electrical betweenness can reflect the influence of the capacity and distribution of generators and load, even quantify the contribution of the nodes and edges to the power flow transmission.

\section{Power system cascading failure power flow analysis based on CNT}

According to the previous analysis, this paper adopts power flow as the load to reflect the distribution of voltage, active power and reactive power at each node and line in the grid network. Over-voltage, under-voltage and overcurrent are considered as boundary conditions of power grid. Once the network failure occurs, the power flow of the grid is calculated again. Time after time, the process is repeated until there is no failure. In addition, connectivity level, global effective performance and percentage of load loss are used to evaluate network performance before and 
after malfunction. Finally, some defense strategies are proposed.

\subsection{Power flow calculation}

The voltage and current in power grid can be obtained through the following power flow calculation equation.

$S=\Gamma(U)$

where $S$ is the given value of the injection power of a certain node; $\Gamma(U)$ is the function of the corresponding impedance of $S$ and node voltage $U$.

\subsection{Voltage and current setting values}

The voltage and current setting values can reflect the boundary values including the upper and lower limits of voltage and current in power system. The detailed description is as:

$U_{\mathrm{c}}=(1+\alpha) U_{0}$

$I_{\mathrm{c}}=(1+\alpha) I_{0}$

where $U_{0}$ and $I_{0}$ are the rated voltage and current; $U_{\mathrm{c}}$ and $I_{\mathrm{c}}$ are the setting values of voltage and current which mean the operating limits of voltage and current of power system; $\alpha(>-1)$ is the tolerance parameter of the network, which represents the capacity of the network to resist interference.

\subsection{Voltage and current constraints}

The power grid has a good operation under the boundary conditions of voltage and current. If power system overvoltage, under-voltage or over-current occurs, the corresponding nodes or lines will be removed.

$U_{\mathrm{c}, \min } \leq U \leq U_{\mathrm{c}, \max }$

$I \leq I_{\mathrm{c}, \max }$

where $U$ and $I$ are the voltage and the current in power system; $I_{\mathrm{c}, \max }$ is the upper operating limit of the current while $U_{\mathrm{c}, \min }$ and $U_{\mathrm{c}, \max }$ are the lower and upper operating limits of the voltage.

\subsection{Network evaluation indicators}

In order to measure the damage of cascading failure in the gird, and evaluate robustness of the power network, three improved indicators are proposed to analyze power system cascading failure.

\section{1) Connectivity level}

Connectivity level is defined as the ratio of the number of nodes in the largest supply area before and after malfunction.
It reflects the connectivity and splitting degree of power system when the failure occurs. The smaller connectivity level indicates the worse robustness of the network.

$g=\frac{N_{G, L}^{\prime}}{N_{G, L}}$

where $N_{G, L}$ and $N_{G, L}^{\prime}$ are the numbers of nodes in the largest connected region which can maintain normal power supply before and after malfunction.

\section{2) Global effective performance}

Global effective performance represents the average tightness between generation nodes and load nodes, and reflects the efficiency and loss of energy in power transmission.

$E=\frac{1}{N_{G} N_{L}} \sum_{i \in G, j \in L} \frac{1}{d_{i j}}$

where subscript $G$ and $L$ are the sets of generation nodes and load nodes; $N_{G}$ and $N_{L}$ are the total numbers of generation nodes and load nodes; $d_{i j}$ is the shortest electrical distance between generation node $i$ and load node $j$.

\section{3) Percentage of load loss}

Percentage of load loss reveals transmission capacity of the whole power grid, which is used to measure the breadth of failure propagation. The more load loss, the greater the damage to the power system.

$p_{\mathrm{PLL}}=\sum_{j \in G_{1}} L_{j} / \sum_{k \in G_{0}} L_{k}$

where $p_{\text {PLL }}$ is the value of percentage of load loss (PLL); subscript $G_{1}$ and $G_{0}$ are the sets of failed and all transmission nodes; $L_{k}$ and $L_{j}$ are the loads of node $k$ and failed node $j$.

\subsection{Calculation process}

Figure 1 shows the flow chart of the process.

1) Calculate the initial power flow in the grid, and the boundary conditions of current and voltage of lines and nodes are expressed as the definition of node capacity $C_{i}=(1+\alpha) L_{i}$.

2) Remove the nodes in the network or wind power integration.

3) Recalculate the power flow in the grid, and take out the nodes or edges whose voltage or current are out-oflimit. Repeat it until there is no failure happened in the network.

4) Evaluate the impacts of network failures on power system depending on evaluation indicators.

5) Propose defense strategies for network failures. 


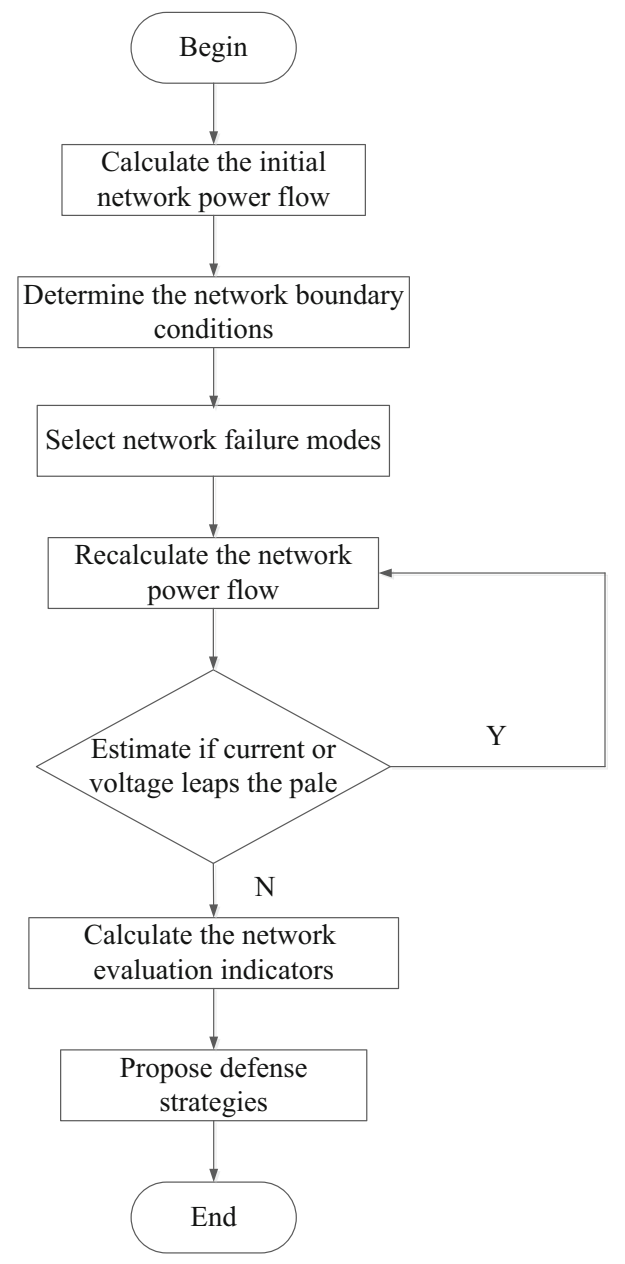

Fig. 1 Flow chart of power grid failure analysis process based on CNT

\section{Case study}

In this paper, an IEEE 30 bus reference power system is utilized to analyze the influence of node removal and wind power on power system. According to the CNT model with wind power integration from Section 2, the IEEE 30 bus system can be described as a weighted, directed and sparse connected graph with 30 nodes and 41 edges, as shown in Fig. 2.

\subsection{Impacts of node removal on power system}

Under the conditions of the fixed voltage and the current boundary, Fig. 3 shows the results by removing all the nodes in order in the network. The average value of connectivity level is 0.6922 . The maximum one of 0.9667 indicates no failure happened after removing a certain node. The minimum one of 0 means the total collapse of the entire network triggered by cascading failure after an important node removal. The average, maximum and

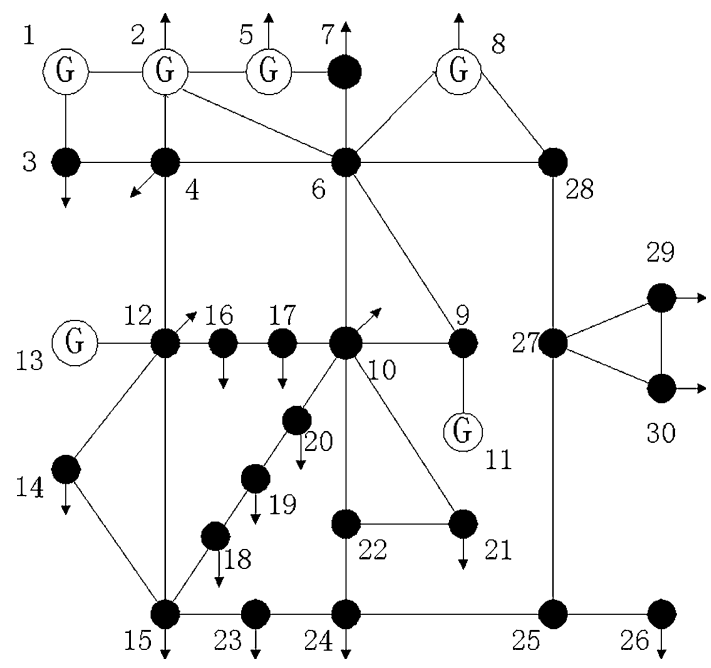

Fig. 2 IEEE 30 bus system topology structure

minimum values of the global effective performance are $1.2345,1.87$ and 0 . When the global effective performance cuts to the minimum, it represents that removing a particular node leads to the interruption of the whole power transmission network. The average value of percentage of load loss is 0.3157 . The maximum value of 1 denotes that all the network loads are lost due to a certain node removal while there is no load loss for the minimum value of 0 . That is, the power grid can maintain the normal operation after taking out a non-significant node.

As can be seen, different node removal will lead to different impacts on the power grid. For instance, the system will not be malfunction when nodes 7 and 17 are removed, while the removal of nodes 1 and 6 will lead to a chain reaction of failures, and even the paralysis of the entire network. Among all the nodes in power system, node 1 has the largest generation capacity, while node 6 has the maximum node degree and electrical betweenness that play an important role in power transmission. To distinguish, the nodes 1 and 6 are considered to be critical nodes [36, 40]. In summary, critical node is an important factor which makes cascading failure occur. To strengthen the protection of critical nodes can reduce the occurrence probability of cascading failures including the advancement of the technics and compatibilizing modification.

\subsection{Impact of wind power on the power system}

1) Comparative analysis of the impact of wind power and node removal

The generation node 13 with smaller generation capacity in IEEE 30 bus system is replaced by a wind farm with the power curve shown in Fig. 4. The comparison results of the impacts of grid-connected wind and node removal in power system are shown in Fig. 5. 

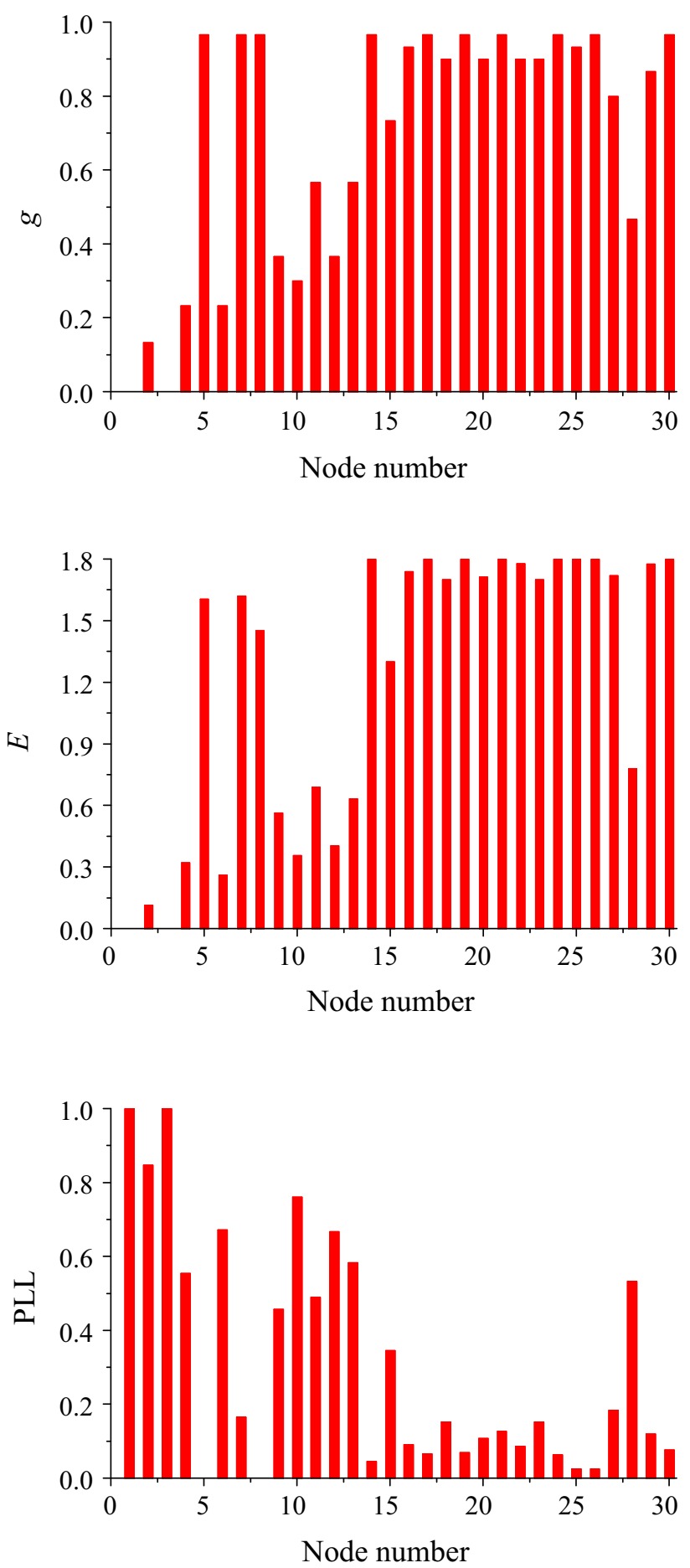

Fig. 3 Results of node number removing all the nodes in order in the network

Figure 5 shows that system evaluation indicators change with the change of the wind power output. Drastic fluctuations of wind power make rapid changes of connectivity level, global effective performance and percentage loss of load. In other words, the more severe power fluctuations,

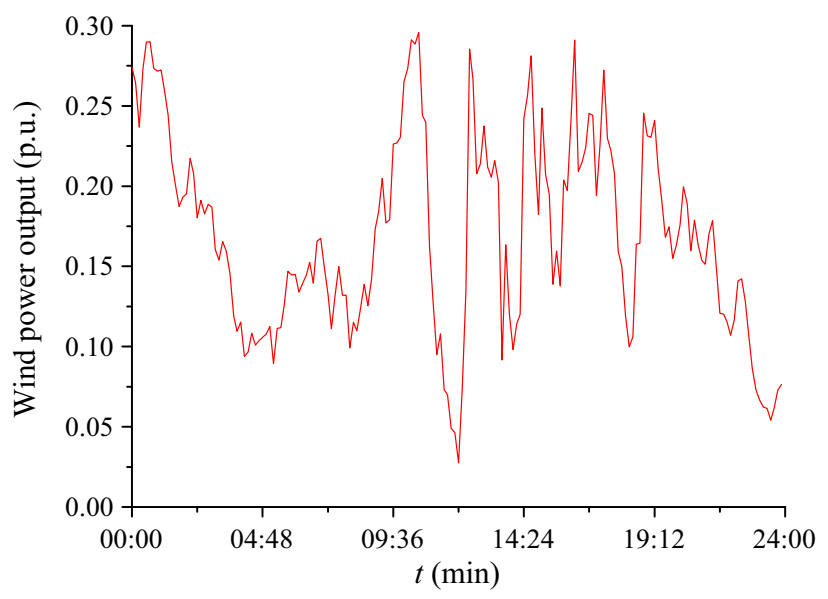

Fig. 4 The power curve of a typical day of a wind farm

the greater the harm of wind power to the system, which can be seen from the time period of 10:00:00 to 11:00:00 and so on. In addition, through the comparisons of wind power integration and node removal, connectivity level and global effective performance of the former ones are $80 \%$ greater than that of the latter. Meanwhile, the percentage of load loss of the former is $80 \%$ less than that of the latter. As a result, the impacts of wind power with certain capacity in power network are less than that of the average of node removal on the same constraints.

2) Impact of grid current tolerance capability

In order to analyze the relationship of boundary conditions of power grid operation and cascading failure, the impact of grid current tolerance capability in the power system is studied.

Supposing that the generation node 13 is replaced by certain capacity of wind farm (Fig. 4), the effects of wind power on the system can be observed from Fig. 6 by adjusting the grid current tolerance capability. It can be seen that the nodes and lines in the grid withstand higher level current, and the impact of wind power weakens with the increase of the current setting coefficient. In other words, with the current setting coefficient increased, the numbers of the failed nodes in the network decrease, and the level of power transmission among nodes is enhanced which can be illustrated by the increase of connectivity level and global effective performance. When the current setting coefficient is over 1.8 , both connectivity level and global effective performance reach their maximum values. That is, there is no failure occurred in the power system. The same conclusions can be obtained for the percentage of load loss. The network load loss decreases with the increase of the grid current tolerance capability. And there is no load dropped out of the power system when the current tolerance capability increases to 1.8 . Thus the 

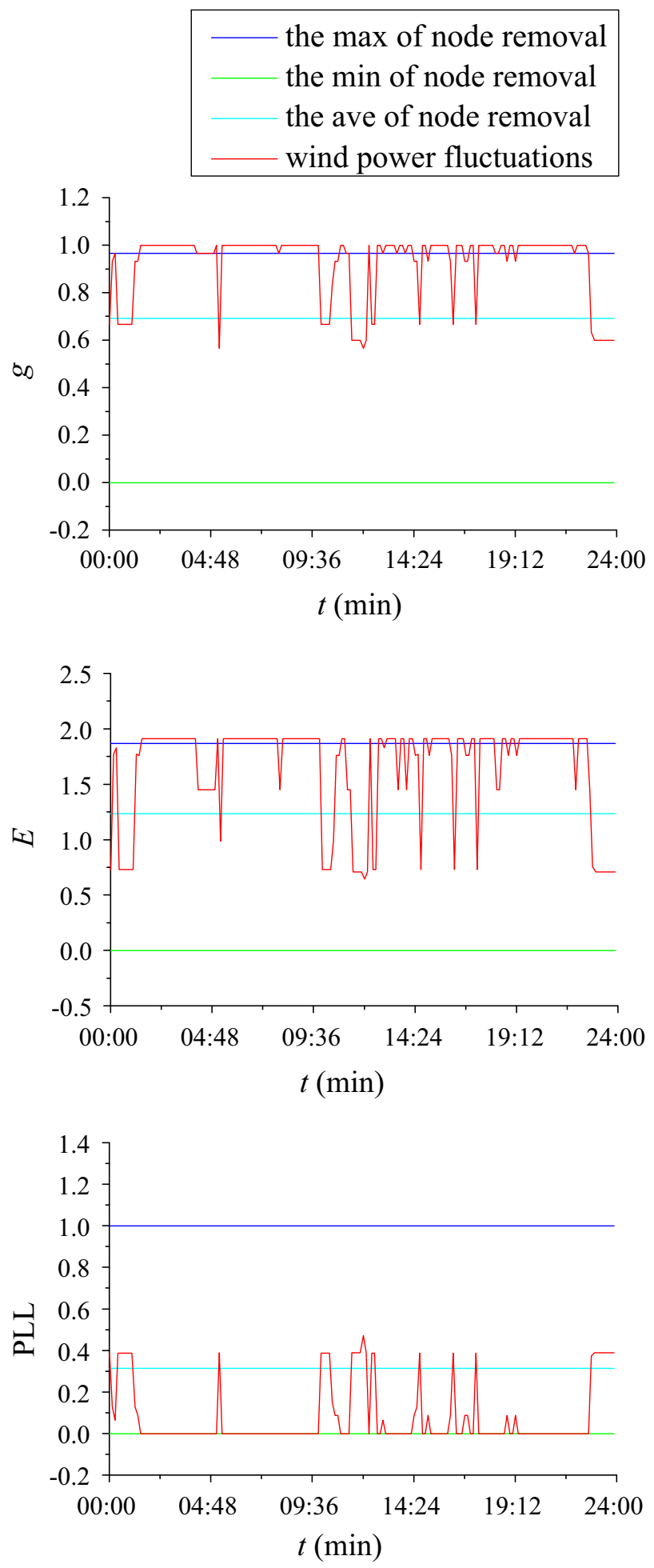

Fig. 5 Contrastive analysis of the impact of wind power and node removal on power system

cascading failure can be reduced and even avoided by the strengthened of grid current tolerance capability through the series current limiting reactor, the upgrading of equipment and lines and so on.
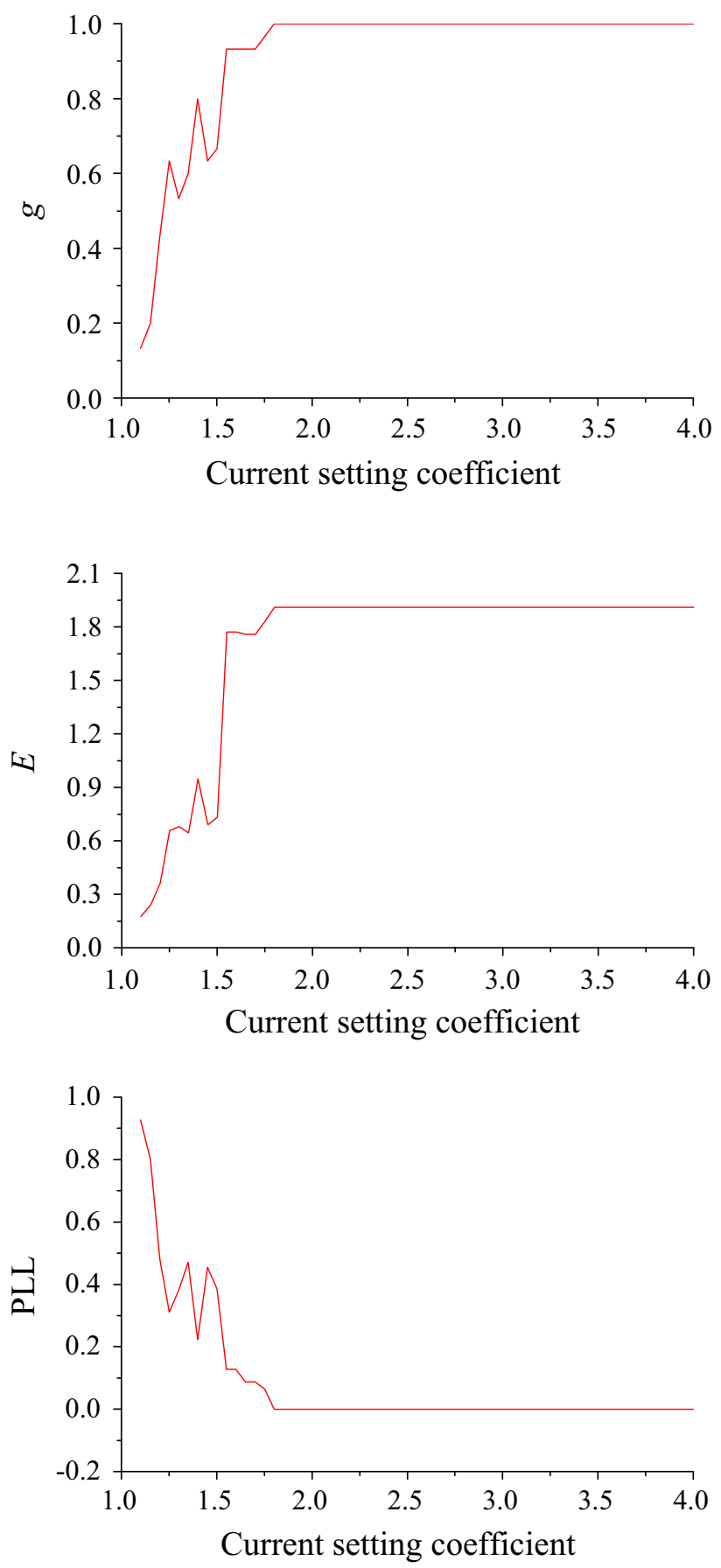

Fig. 6 Impacts of grid current tolerance capability change on power system

3) Impact of wind power instantaneous penetration

The instantaneous penetration of wind power can be described as the ratio of the present wind power production to the present system load [41].

Displaced the traditional generator with wind farm (Fig. 4) in node 13, Fig. 7 illustrates the simulation results of connectivity level, global effective performance and 

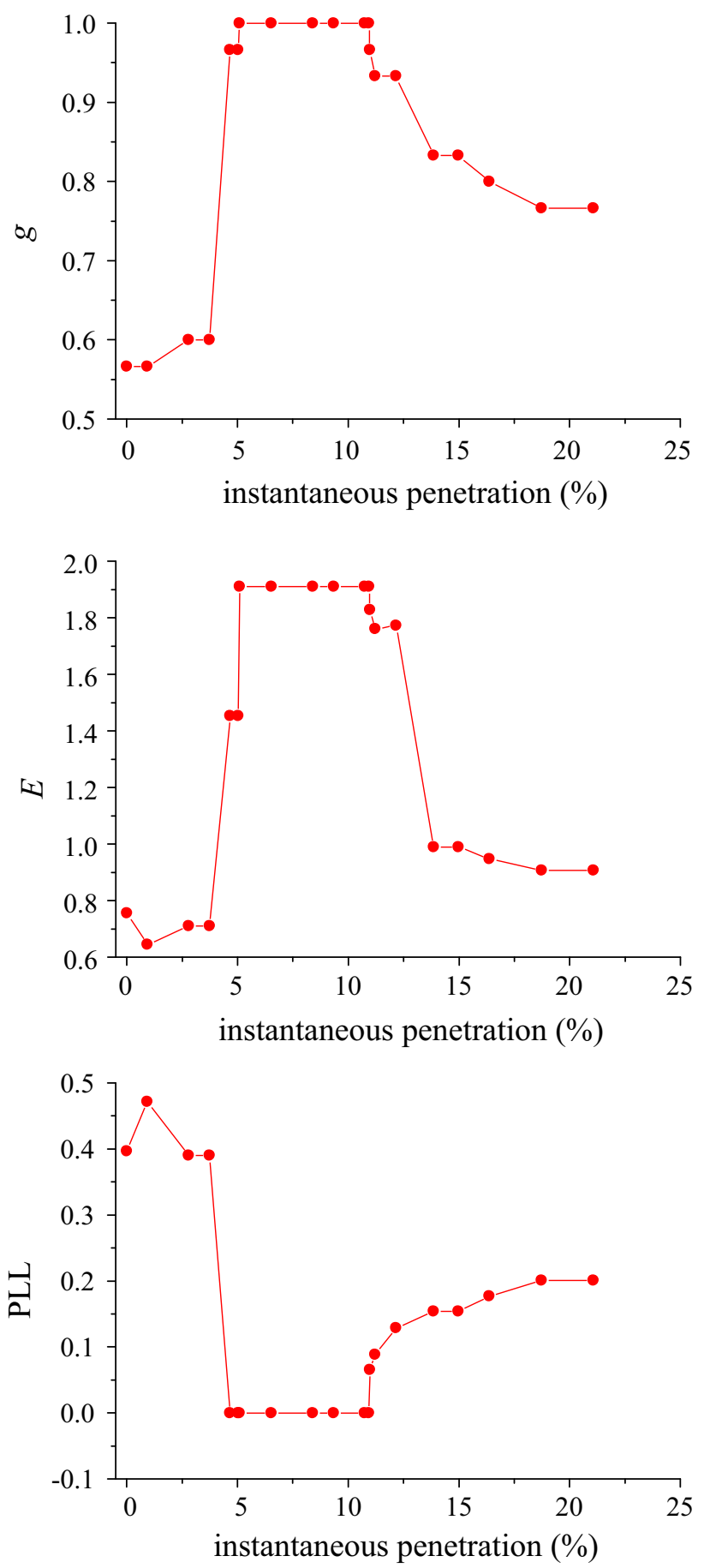

Fig. 7 Impact of wind power instantaneous penetration on power system

percentage of load loss respectively with wind power instantaneous penetration of $0 \%, 0.93 \%, 2.79 \%, 3.72 \%$, $4.66 \%, 5.03 \%, 5.08 \%, 6.53 \%, 8.40 \%, 9.34 \%, 10.74 \%$, $10.93 \%, 10.98 \%, 11.21 \%, 12.15 \%, 13.86 \%, 14.97 \%$, $16.37 \%, 18.72 \%$, and $21.06 \%$.

When the wind power instantaneous penetration is lower, connectivity level and global effective performance That is to say, the low output power of a certain wind farm will deteriorate the security operation of power system. With the output power of the wind farm increasing, connectivity level and global effective performance increase and the percentage of load loss decreases. When the

Fig. 8 Impacts of different load points of wind power integration on power system 

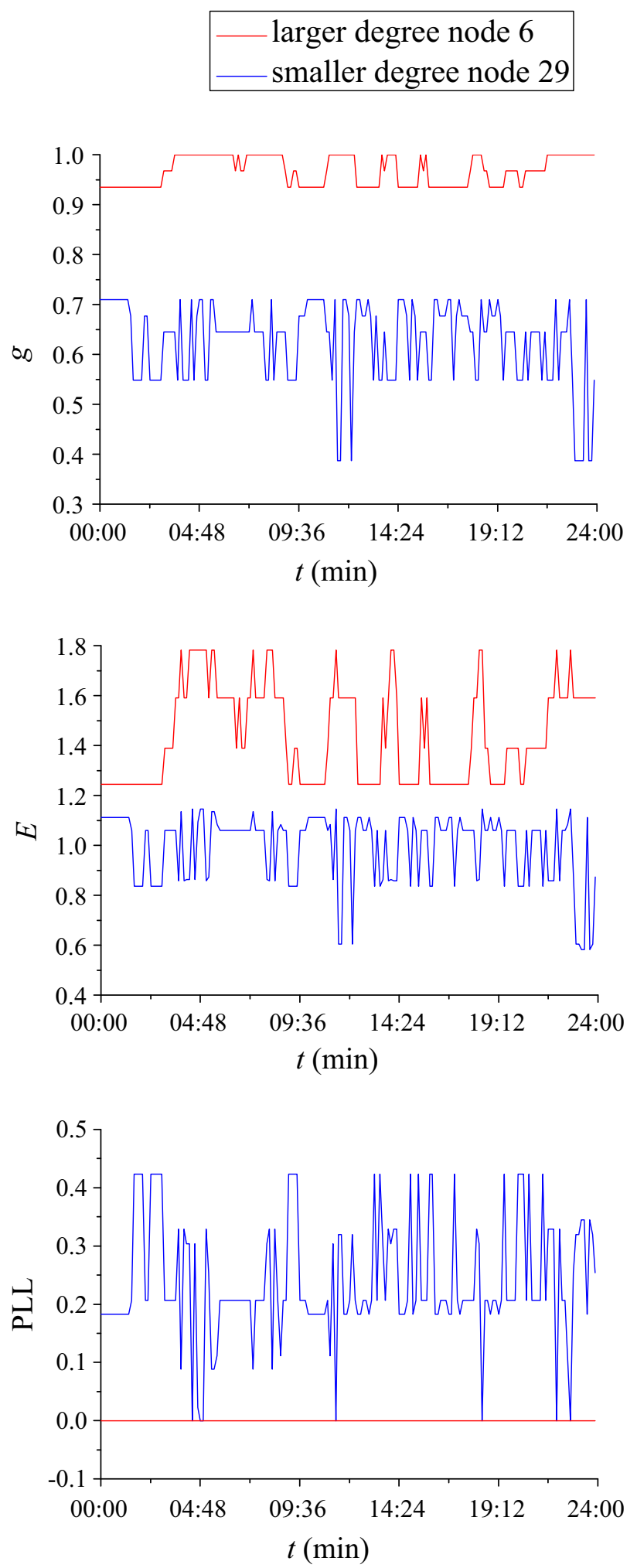

Fig. 9 Impacts of different degree points of wind power integration on power system

instantaneous penetration of wind power locates in the range of $5.08 \%-10.93 \%$, both connectivity level and global effective performance reach the maximum values while percentage of load loss reduced to the minimum of zero. Power system in this situation is also robust enough with little chance of cascading failure.

However, when wind power instantaneous penetration grows still higher to $21.06 \%$, connectivity level and global effective performance of the network decrease, while the percentage of load loss increases. This reflects that the power system becomes more vulnerable to cascading failure with the increase of wind power instantaneous penetration.

It can be seen from the above simulations that different wind power instantaneous penetrations have different impacts on power system performance. Therefore, proper wind power install capacity plan is important for the reliable operation of power network. What's more, wind farm active power control, such as the utilization of energy storage, can mitigate nodes or line failures and then the possibility of cascading failure.

4) Impacts of wind power coupling locations

To analyze the impacts of different points of wind power coupling with the power grid, a typical wind farm in north China (Fig. 4) is added as the 31 st power node in the IEEE 30 bus system, where the locations of the wind farm is set at the nodes with rather larger load or degree, respectively.

It can be seen from Fig. 8 and Fig. 9 that the larger degree and load nodes of the points of wind farm integration, the better of connectivity level and global effective performance, as well no load loss in the power system. Therefore, the selection of greater load and degree nodes for wind farm coupling point has less influence on power system performance.

\section{Conclusion}

With the development of complex network theory, it provides a new perspective to study and analyze cascading failures which will be deteriorated with the increasing of wind power instantaneous penetration. In this paper, improved CNT network topology principles and methods considering wind farms integration are presented. A cascading failure power flow analysis model is established with three typical evaluation indicators including connectivity level, global effective performance and percentage of load loss. The IEEE 30 bus system is taken as an example to investigate comparatively the influence of node attacks and wind power integration in the power system, as well grid current tolerance capability, wind power instantaneous penetrations and wind farm grid coupling points in the power grid. Some key conclusions can be obtained as follows: 
1) The failures of the critical nodes will lead to a series of chain reaction, and strengthening the protection of these nodes can reduce the occurrence probability of cascading failure due to wind power integration.

2) The normal operation of power system is subject to grid current tolerance capability, and cascading failure can be reduced and even avoided by the strengthened of grid current tolerance capability.

3) The instantaneous penetration of wind power affects system cascading failure performance, while appropriate wind power install capacity and active power control can reduce or avoid cascading failure probability.

4) The coupling point of wind power with power system is sensitive to cascading failure, while the selecting of larger degree and heavy load nodes for wind power is conducive to system stable operation.

Acknowledgments This work was financially supported by a grant from the National Basic Research Program of China (973 Program) (No. 2012CB215204), and the Key Project of the CAS Knowledge Innovation Program "Research and demonstration of the coordinated control system based on multi-complementary energy storage" (No. KGCX2-EW-330).

Open Access This article is distributed under the terms of the Creative Commons Attribution License which permits any use, distribution, and reproduction in any medium, provided the original author(s) and the source are credited.

\section{References}

[1] Bak P, Tang C, Wiesenfeld K (1987) Self-organized criticality: an explanation of $1 / f$ noise. Phys Rev Lett 59(4):381-384

[2] U.S.-Canada power system outage task force (2004) Final report on the August 14, 2003 blackout in the United States and Canada: causes and recommendations

[3] Gan DQ, Hu JY, Han ZX (2004) Thoughts for some international blackout in 2003. Autom Electr Power Syst 28(3):1-4, 9 (in Chinese)

[4] Deng HQ, Ai X, Zhao L (2006) Grid cascading failure analysis review. Modern electric power 23(6):10-20 (in Chinese)

[5] Sun K, Han ZX, Cao YJ (2005) Comments on complex grid cascading failure model. Power Syst Technol 29(13):1-9 (in Chinese)

[6] Lu ZX (2005) Reliability research of the grid complexity and blackouts. Autom Electr Power Syst 29(12):93-97 (in Chinese)

[7] Zhan Y, Cheng HZ, Xiong HG (2005) Review of the grid cascading failure. Electr Power Autom Equip 25(9):93-98 (in Chinese)

[8] Jonnavithula S, Billinton R (1997) Topological analysis in bulk power system reliability evaluation. IEEE Trans Power Syst 12(1):456-463

[9] Solé RV, Rosas-Casals M, Corominas-Murtra B et al (2008) Robustness of the European power grids under intentional attack. Phys Rev E 77(2):026102/1-026102/7

[10] Holmgren A (2006) Using graph models to analyze the vulnerability of electric power networks. Risk Anal 26(4):955-969
[11] Rosato V, Bologna S, Tiriticco F (2007) Topological properties of high-voltage electrical transmission networks. Electr Power Syst Res 77(2):99-105

[12] Holme F, Kim BJ (2002) Vertex overload breakdown in evolving networks. Phys Rev E 65(6):066109/1-066109/8

[13] Kim BJ, Yoon CN, Han SK (2002) Attack vulnerability of complex networks. Phys Rev E 65(5):056109/1-056109/14

[14] Lai YC, Motter AE, Nisllikawa T (2004) Attacks and cascades in complex networks. Lect Notes Phys 650:299-310

[15] Motter AE, Lai YC (2002) Cascade-based attacks on complex networks. Phys Rev E 66(6):065102/1-065102/4

[16] Latora V, Marchiori M (2001) Efficient behavior of small-world networks. Phys Rev Lett 87(19):198701/1-198701/4

[17] Crucitti P, Latora V, Marchiori M (2004) Model for cascading failures in complex networks. Phys Rev E 69(4):045104/ $1-045104 / 4$

[18] Wang JW, Rong LL, Zhang L (2009) A model for cascading failures in complex networks with a tunable parameter. Mod Phys Lett B 23(40):1323-1332

[19] Wang JW, Rong LL (2009) Cascade-based attack vulnerability on the US power grid. Saf Sci 47(10):1332-1336

[20] Wang WX, Chen GR (2008) Universal robustness characteristic of weighted networks against cascading failure. Phys Rev E 77(2):026101/1-026101/5

[21] Wang JW, Rong LL (2009) Vulnerability of effective attack on edges in scale-free networks due to cascading failures. Int J Mod Phys C 20(8):1291-1298

[22] Cotilla-Sanchez E, Phines PDH, Barrows C et al (2012) Comparing the topological and electricalstructure of the North American electric power infrastructure. IEEE Syst J 6(4):616-626

[23] Arianos S, Bompard E, Carbone A et al (2009) Power grid vulnerability: a complex network approach. Chaos 19:013119/ $1-013119 / 6$

[24] Bompard E, Wu D, Xue F (2011) Structural vulnerability of power systems: a topological approach. Electr Power Syst Res 81(7):1334-1340

[25] Bompard E, Masera M, Napoli R et al (2009) Assessment of structural vulnerability for power grids by network performance based on complex networks. In: Critical information infrastructure security, LNCS 5508: Proceedings of the 3rd international workshop on critical information infrastructures security (CRITIS'08). Rome, Italy, 13-15 Oct 2008, pp 144-154

[26] Xu L, Wang XL, Wang XF (2010) The application of betweenness in the critical line identification of power system. Proc CSEE 30(1):33-39 (in Chinese)

[27] Xu L, Wang XL, Wang XF (2010) Propagation mechanisms and effective defense of power system cascading failure based on betweenness. Proc CSEE 30(13):61-67 (in Chinese)

[28] Wang K, Zhang BH, Zhang Z et al (2011) An electrical betweenness approach for vulnerability assessment of power grids considering the capacity of generators and load. Phys A 390(23/24):4692-4701

[29] Bompard E, Pons E, Wu D (2012) Extended topological metrics for the analysis of power grid vulnerability. IEEE Syst J 6(3):481-487

[30] Mohsenian-Rad AH, Leon-Garcia A (2011) Distributed internetbased load altering attacks against smart power grids. IEEE Trans Smart Grid 2(4):667-674

[31] Salmeron J, Wood K, Baldick R (2004) Analysis of electric grid security under terrorist threat. IEEE Trans Power Syst 19(2):905-912

[32] Albert R, Jeong H, Barabási AL (2000) Error and attack tolerance of complex networks. Nature 406:378-382

[33] Yu K, Rong LL, Wang JW (2009) A new attack on scale-free networks based on cascading failures. Mod Phys Lett B 23(20/ 21):2497-2505 
[34] Wu ZX, Peng G, Wang WX et al (2008) Cascading failure spreading on weighted heterogeneous networks. J Stat Mech 5:P05013/1-P05013/14

[35] Li P, Wang BH, Sun $\mathrm{H}$ et al (2008) A limited resource model of fault-tolerant capability against cascading failure of complex network. Eur Phys J B 62(1):101-104

[36] Su HL, Li Y (2012) Exploring the vulnerability of the components from the complex network characteristics of power system. Autom Electr Power Syst 36(23):12-17 (in Chinese)

[37] Sun K (2005) Complex networks theory: a new method of research in power grid. In: Proceedings of the 2005 IEEE/PES transmission and distribution conference and exhibition: Asia and Pacific, Dalian, China, 14-18 Aug 2005, 6 pp

[38] Cao YJ, Chen XG, Sun K (2006) Identification of vulnerable lines in power grid based on complex network theory. Electr Power Autom Equip 26(12):1-5, 31 (in Chinese)

[39] Saedy M, Kelley B (2012) Foundations of ultra-low power scale free sensor networks for cluster to cluster communications. IEEE Sens J 12(9):1890-2881
[40] Chen XG, Sun K, Cao YJ et al (2007) Identification of vulnerable lines in power grid based on complex network theory. In: Proceedings of the 2007 IEEE Power Engineering Society general meeting, Tampa, FL, USA, 24-28 Jun 2007, 6 pp

[41] Tarnowski GC (2011) Coordinated frequency control of wind turbines in power systems with high wind power penetration. $\mathrm{PhD}$ Thesis, Technical University of Denmark

Yushu SUN works as a research assistant at the Institute of Electrical Engineering of Chinese Academy of Sciences. His research interests include renewable energy generation, energy management platform and hybrid energy storage coordinated control in power system.

Xisheng TANG is currently a professor at the Institute of Electrical Engineering of Chinese Academy of Sciences. His research interests are in power system stability and control, distributed generation and energy storage technologies, application of power electronics in power system. 